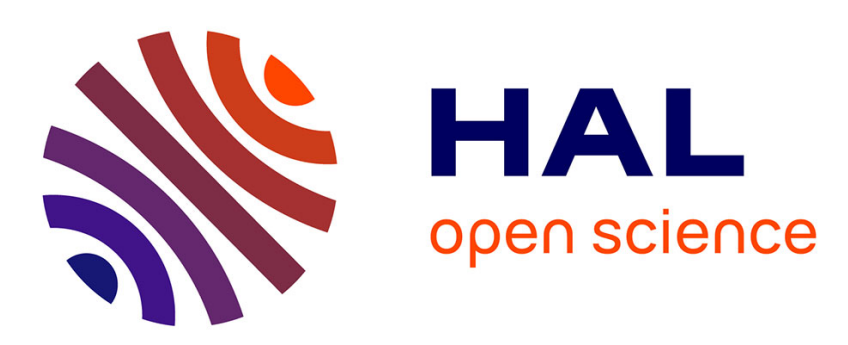

\title{
Coherent turbulent structures at the mixing-interface of a square open-channel lateral cavity
}

\author{
Emmanuel Mignot, Wei Cai, Gaby Launay, Nicolas Rivière, Cristian
}

Escauriaza

\section{- To cite this version: \\ Emmanuel Mignot, Wei Cai, Gaby Launay, Nicolas Rivière, Cristian Escauriaza. Coherent turbulent structures at the mixing-interface of a square open-channel lateral cavity. Physics of Fluids, 2016, 28 (4), pp.045104. 10.1063/1.4945264 . hal-01516083}

\section{HAL Id: hal-01516083 \\ https://hal.science/hal-01516083}

Submitted on 7 Mar 2018

HAL is a multi-disciplinary open access archive for the deposit and dissemination of scientific research documents, whether they are published or not. The documents may come from teaching and research institutions in France or abroad, or from public or private research centers.
L'archive ouverte pluridisciplinaire HAL, est destinée au dépôt et à la diffusion de documents scientifiques de niveau recherche, publiés ou non, émanant des établissements d'enseignement et de recherche français ou étrangers, des laboratoires publics ou privés. 


\section{Coherent turbulent structures at the mixing-interface of a square open- channel lateral cavity}

Emmanuel Mignot, Wei Cai, Gaby Launay, Nicolas Riviere, and Cristián Escauriaza

Citation: Physics of Fluids 28, 045104 (2016); doi: 10.1063/1.4945264

View online: https://doi.org/10.1063/1.4945264

View Table of Contents: http://aip.scitation.org/toc/phf/28/4

Published by the American Institute of Physics

\section{Articles you may be interested in}

Surface velocity divergence model of air/water interfacial gas transfer in open-channel flows Physics of Fluids 29, 045107 (2017); 10.1063/1.4981228

Are there two regimes in strongly rotating turbulence?

Physics of Fluids 28, 045103 (2016); 10.1063/1.4944878

Numerical and experimental investigation of natural flow-induced vibrations of flexible hydrofoils Physics of Fluids 28, 075102 (2016); 10.1063/1.4954785

The roll-up and merging of coherent structures in shallow mixing layers Physics of Fluids 28, 094103 (2016); 10.1063/1.4960391

Impact of domain size and statistical errors in simulations of homogeneous decaying turbulence and the Richtmyer-Meshkov instability

Physics of Fluids 28, 045106 (2016); 10.1063/1.4944877

On the validity of the quasi-steady-turbulence hypothesis in representing the effects of large scales on small scales in boundary layers

Physics of Fluids 28, 045102 (2016); 10.1063/1.4944735

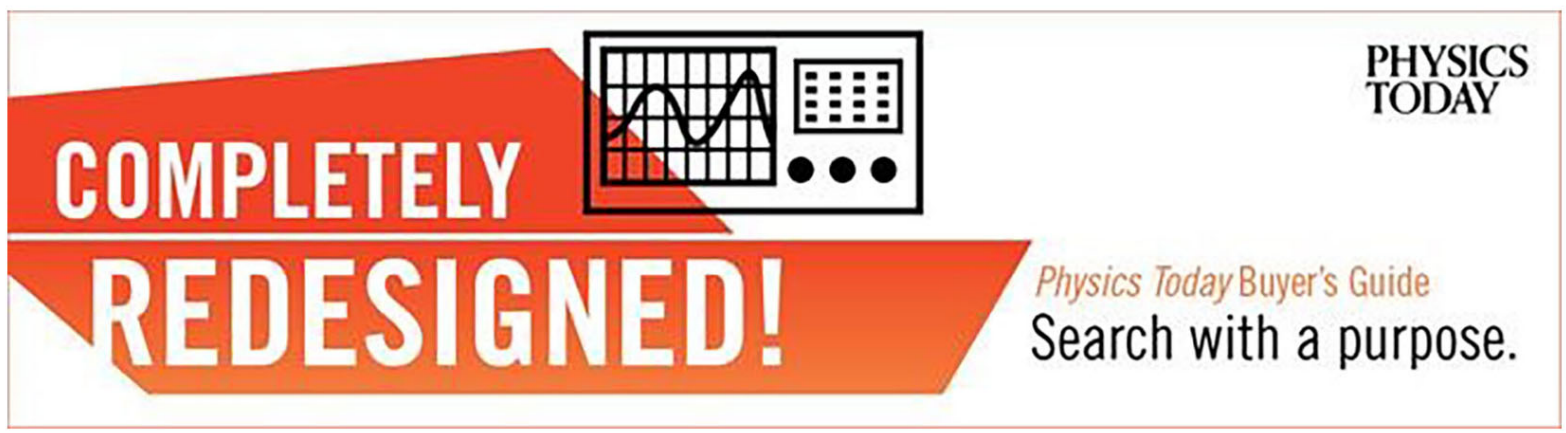




\title{
Coherent turbulent structures at the mixing-interface of a square open-channel lateral cavity
}

\author{
Emmanuel Mignot, ${ }^{1, a}$ ) Wei Cai, ${ }^{1}$ Gaby Launay, ${ }^{1}$ Nicolas Riviere, ${ }^{1}$ \\ and Cristián Escauriaza ${ }^{2}$ \\ ${ }^{1}$ Univ Lyon, INSA Lyon, CNRS, LMFA UMR5509, F-69621 Villeurbanne, France \\ ${ }_{2}^{2}$ Departamento de Ingeniería Hidráulica y Ambiental, Pontificia Universidad Católica de \\ Chile, Av. Vicuña Mackenna 4860, 7820436 Santiago, Chile and Centro de Investigación para \\ la Gestión de Desastres Naturales (CIGIDEN), Santiago, Chile
}

(Received 15 October 2015; accepted 21 March 2016; published online 4 April 2016)

\begin{abstract}
The self-sustained turbulent shear or mixing layer that develops at the interface between a channel and a lateral cavity is the leading mechanism that drives the transfer of momentum and mass in these open-channel flows. Therefore, quantifying the interactions between large-scale vortical structures and the enhanced velocity fluctuations at the interface is critical to understand the physical processes which control the exchanges between the cavity and the main channel. In this investigation, we carry out hydrodynamic experiments in a straight, rectangular channel with a lateral square cavity. We measure the velocity field in a horizontal plane using particle image velocimetry to study the dynamics and statistics of the mixing layer, including the effects of the adverse pressure gradient at the downstream corner. By combining proper-orthogonal decomposition with a vortex identification technique, we investigate the motion of coherent structures and calculate the histograms of their trajectories, capturing also additional phenomena such as the vortex splitting, and the interaction of the mixing layer with inner vortices formed inside the cavity. We finally quantify the mass transport capacity of the mixing layer, from the statistics of the transverse velocity at the interface. (C) 2016 AIP Publishing LLC. [http://dx.doi.org/10.1063/1.4945264]
\end{abstract}

\section{INTRODUCTION}

Turbulent flows past a cavity are encountered in many problems of significant interest in aerodynamics and hydrodynamics, including flow-induced vibrations, industrial manufacturing, and acoustics. ${ }^{1-3}$ They have also great relevance in many environmental flows, as cavities form surface-storage zones in rivers and streams, controlling in many cases the sediment transport, nutrient uptake, and contaminant transport processes in these aquatic environments. ${ }^{4-6}$

Cavity flows are characterized by complex flow features such as quasi-periodic vortices produced at the interface between the main flow and the cavity, multiple recirculating zones, coherent vortical structures in the cavity that interact with each other and with the walls, development of centrifugal instabilities due to streamline curvature, etc. ${ }^{7-10}$ These flow features have been extensively studied in experiments with rectangular geometries, providing descriptions of the flow topology and the dynamics of the coherent structures in the vicinity and inside the cavity. The leading mechanism in the dynamics of the flow is the emergence of a self-sustained shear layer that develops from the upstream corner of the cavity where the vortices are shed to the downstream corner where the vortices impinge and split between both regions. These phenomena are accompanied by additional processes that occur at different time scales such as low-frequency flapping of the entire shear-layer and vorticity fluxes from the wall due to streamwise pressure gradients downstream of the cavity. ${ }^{10}$

Depending on the aspect ratio of the cavity, the flow is characterized by the formation of one or more large-scale vortical structures that occupy almost the entire cavity volume, in addition to

a)emmanuel.mignot@insa-lyon.fr 
corner structures that form due to vortex-wall interactions. Most of the cavity flows documented in the literature have focused on two-dimensional (2D) geometries in an approaching turbulent boundary layer flow, where the cavity configuration corresponds to a slit in the wall, without influence of lateral constrains. In river flows and more generally in open-channel hydraulics, however, lateral cavities are open to the atmosphere on one face and constricted by the bed on the opposite face, forming a three-dimensional (3D) geometry. They either correspond to open lateral cavities ${ }^{4,9,11}$ or to the so-called groyne fields. ${ }^{12-14}$ Recent experimental investigations have turned their attention to lateral cavities in open-channel flows. ${ }^{9,11,15}$ The specific characteristics of the shear layer in this type of flows, however, have not yet been described in detail even though the vortex interactions and large-scale velocity fluctuations constitute the underlying physical mechanisms that control mass and momentum transport across the interface.

To further our understanding on the interactions of the coherent vortices at the interface between a channel and a lateral cavity and their consequences on mass exchange, we perform experiments in an open lateral cavity flow configuration, in a rectangular channel without free-surface oscillations (i.e., under no-seiching conditions). We focus on the motion and the statistical characterization of vortical structures of the shear-layer with the following objectives: (1) understand the momentum exchange mechanisms at the interface by analyzing the instantaneous flow field, the mixing layer expansion rate, and the fundamental frequency of the flow dynamics. We focus our attention on the impact of the adverse pressure gradient generated at downstream corner of the cavity; (2) describe the mechanisms of vortex shedding and propagation and their consequences in terms of the transverse velocity fluctuations that are produced by the coherent structures of the shear-layer; and (3) quantify the mass transport capacity of the mixing layer, based on the transverse velocity across the interface.

The paper is organized as follows: In Section II we present the description of the experimental setup and measurement methodologies with a two-dimensional particle image velocimetry (2D-PIV) system. Subsequently, in Section III we discuss the mean flow and turbulence statistics in the cavity and the shear layer. We later quantify the dynamics of the coherent turbulent structures and flow oscillations in the shear-layer in Section IV. In Section V we estimate the mass exchange coefficient across the mixing layer from the velocity field. Finally, in the conclusions of Section VI, we summarize the findings of this investigation and discuss future research possibilities.

\section{EXPERIMENTAL SETUP}

Experiments are performed at the Laboratoire de Mecanique des Fluides et d'Acoustique at the Universite de Lyon. The facility consists of a $4.9 \mathrm{~m}$ long and $b=0.3 \mathrm{~m}$ wide horizontal glass channel of rectangular cross section, connected with a square cavity of width and length equal to $b$ on the side of the channel at mid-length, as depicted in Figure 1. The inlet discharge in the channel is fixed to $Q=0.0035 \mathrm{~m}^{3} / \mathrm{s}$, measured using a Promag 50 flowmeter (from Endress Hauser; with an accuracy of $5 \times 10^{-5} \mathrm{~m}^{3} / \mathrm{s}$ ) within the pumping loop. A honeycomb mesh is installed at the inlet of the channel, which serves to stabilize and straighten the inflow, and a sharp crested weir at the outlet is used to fix the water depth to $h=0.07 \mathrm{~m}( \pm 0.15 \mathrm{~mm})$ at the entrance of the cavity. The bulk velocity in the main channel is therefore equal to $U_{b}=Q /(b h)=0.166 \mathrm{~m} / \mathrm{s}$, such that the Reynolds number of the flow, expressed in terms of the hydraulic diameter of the channel $D_{h}$ and the kinematic viscosity $v$, is defined as $R e=U_{b} D_{h} / v=4 Q /[v(b+2 h)]=3.2 \times 10^{4}$. The Froude number is thus written as follows, $F r=U_{b} /(g h)^{0.5}=0.2$. It was verified that the maximum free-surface oscillations in the cavity remained negligibly small, with an amplitude smaller than $1 \%$. More details on the experimental setup can be found in previous investigations. ${ }^{16,17}$

The time-resolved velocity field was measured in a horizontal plane, collecting the $u$ and $v$ components of the velocity field along the cartesian $x$ and $y$ axes, respectively, as shown in Figure 1 . We perform our analysis using PIV at the plane located at an elevation of $z_{L}=0.05 \mathrm{~m}$, that is, $z_{L} / h=0.71$, as indicated in Figure 1, which is sufficiently far from the bed to expect any dependency to the measurement elevation. ${ }^{9}$ Polyamid particles with a diameter of $50 \mu \mathrm{m}$ are added to the water, and we employ a $40 \mathrm{~mW}$ continuous laser coupled with a cylindrical lens that generates a $1 \mathrm{~mm}$ horizontal light layer at the elevation of the plane. Our focus is the section of the flow that comprises 


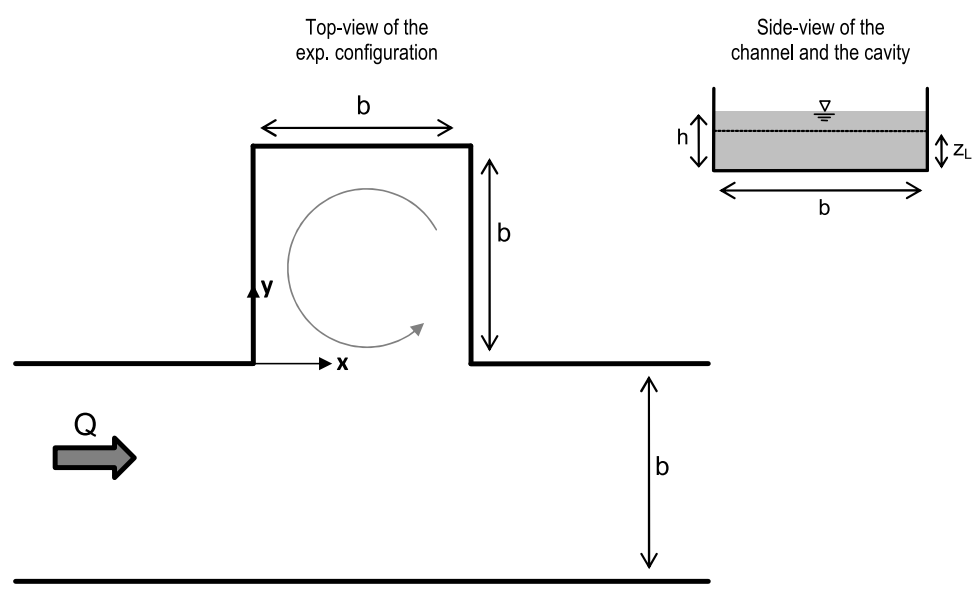

FIG. 1. Experimental configuration of the channel and lateral cavity with rectangular cross sections, where $b=0.3 \mathrm{~m}$ and the water depth is $h=0.07 \mathrm{~m}$. The PIV is performed in a plane at an elevation $z_{L}=0.05 \mathrm{~m}$.

the shear layer, at the interface of the main channel with the cavity, as shown in Figure 1. In this region, the images are captured with a video camera located above the cavity, which records the motion of the particles for over 3000 consecutive images, using a resolution of $1280 \times 960$ pixels with an average spatial resolution of $0.3 \mathrm{~mm}$ per pixel at a frequency equal to $30 \mathrm{~Hz}$. The commercial software Davis (from Lavision) allows us to correct any image distortion, subtract the background from the images and compute the instantaneous velocity fields with a spatial resolution of $7 \mathrm{~mm}$, i.e., 43 measurement points per cavity length $b$. For the estimation of the uncertainty in the velocity measurements, we first verify that the Stokes number associated to the seeding particles remains very low to ensure that the particles follow closely the flow. Following previous work on PIV measurements, ${ }^{17,18}$ the Gaussian peak fitting process generates an uncertainty of the estimated flow displacement equal to 0.1 pixel, which should be added to other sources of uncertainty, mainly due to seeding concentration gradients in the flow field (see Jahanmiri, ${ }^{18}$ for details). Since these gradients are insignificant in the present experiment, it is possible to assume that this second source of error leads to an uncertainty of about the same magnitude as for the peak fitting process. In the present experiment, this leads to a final uncertainty of the instantaneous velocity estimation about equal to $1.8 \mathrm{~mm} / \mathrm{s}[2 \times 0.1 \times$ spatial resolution $(0.3 \mathrm{~mm} /$ pix $) \times$ acquisition frequency $(30 \mathrm{~Hz})]$. Note that we additionally compared the velocity magnitude and fluctuations in the mixing layer with those measured using an Acoustic Doppler Velocimeter, which exhibited a good agreement with the PIV measurements.

\section{MEAN FLOW DESCRIPTION}

We begin the presentation and discussion of the velocity field in the shear layer region by focusing on the time-averaged and statistical features of the 2D flow on the measurement plane. As indicated by Tuna et al., ${ }^{9}$ the mean flow in the cavity is mostly two-dimensional, except in the region closer to the bed. In Figure 2 we plot the mean streamlines and contours of the velocity magnitude in the cavity, showing that one recirculation cell occupies almost the entire available volume, as seen in previous investigations for similar flows. ${ }^{19-21}$

Figure 2 shows that the velocities in the outer layer of the recirculating cell in the cavity have an approximate magnitude of $0.025 \mathrm{~m} / \mathrm{s}$, which corresponds to $0.15 U_{b}$, similar to the ratio found by Tuna et al. ${ }^{9}$ in their recent experiments. The shear or mixing layer is formed by the velocity gradient that is generated across the interface of the main channel with the cavity. ${ }^{22,23}$ To show the magnitude of this velocity difference, the zoomed images plotted in Figure 3 depict the approaching velocity profile in the main channel upstream from the cavity, and the streamlines and velocity contours inside the cavity at the location of the shear layer. The high-velocity flow in the channel has a maximum streamwise velocity located at $y / b=-0.3$, and appears to evolve gradually in the streamwise direction from $x / b=-1.33$ to -0.66 as observed in Figure 3(a). We report the development of the upstream channel 


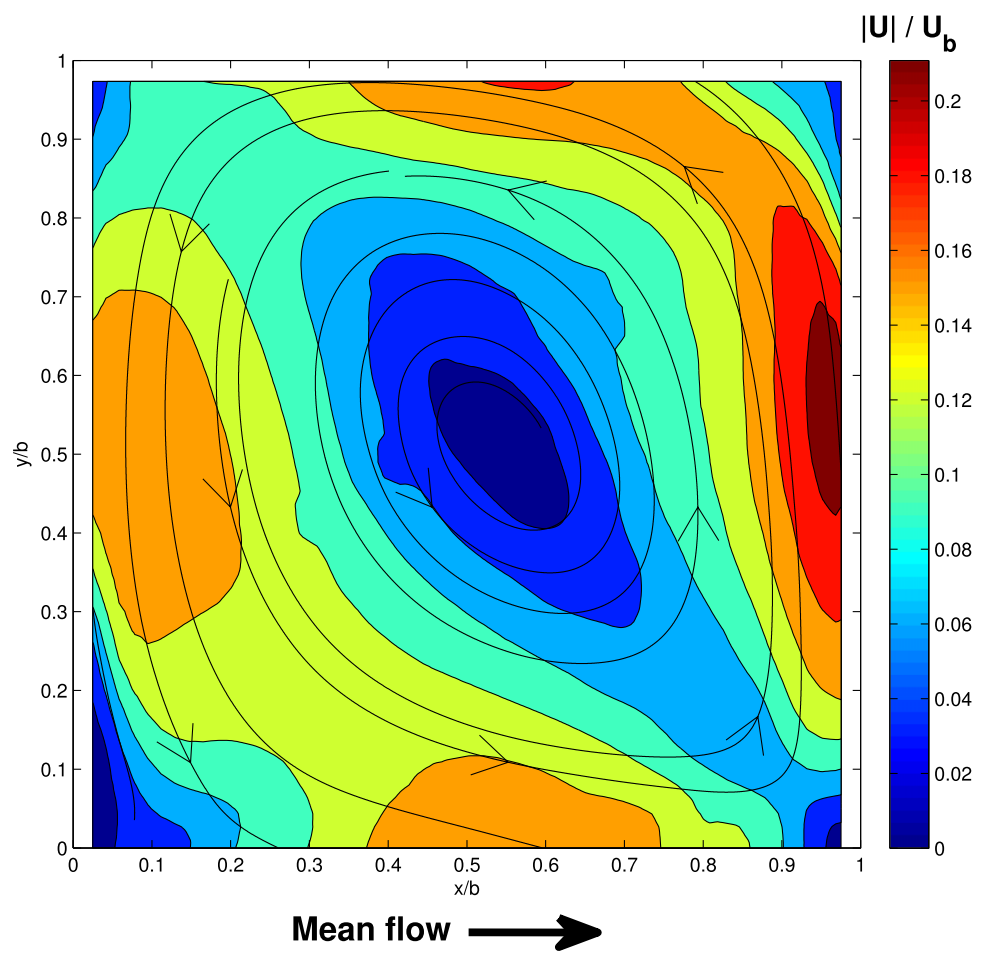

FIG. 2. Time averaged flow field at the horizontal plane, showing the contours of the non-dimensional velocity magnitude inside the cavity. A large asymmetric vortical structure with a vertical axis occupies most of the cavity volume.

flow at the sidewall, since the boundary layer thickness is one of the parameters that characterizes the flow past lateral cavities. This information is therefore important to reproduce and compare the present cavity flow with other similar flow fields.

To study the downstream evolution of the shear layer along the interface, we plot in Figure 4 the time-averaged streamwise non-dimensional velocity profiles and the transverse mean velocity gradients at the interface, denoted as $\bar{u} / U_{b}$ and $\frac{b}{U_{b}} \frac{\partial \bar{u}}{\partial y}$, respectively. The measurements show that the flow has many similarities with free mixing layers that appear between two parallel streams, ${ }^{22,23}$ and with real-life flows where the axis of the mixing layer is geometrically imposed, i.e., compound channels where the streamwise axis is constrained along the bank between both channels,${ }^{24}$ and vegetated or urban canopies where the axis is constrained along the crest of the canopy. ${ }^{25}$ Figure 4(a) shows the development of the mean streamwise velocity profile at the interface, as the momentum is transferred inside the cavity and the velocities increase for $y / b>0$ in the downstream direction, due to flow

(a)

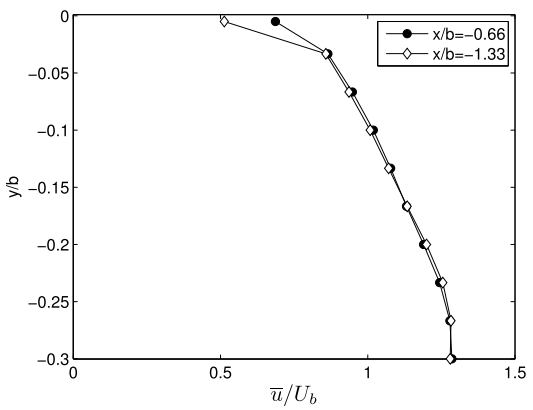

(b)

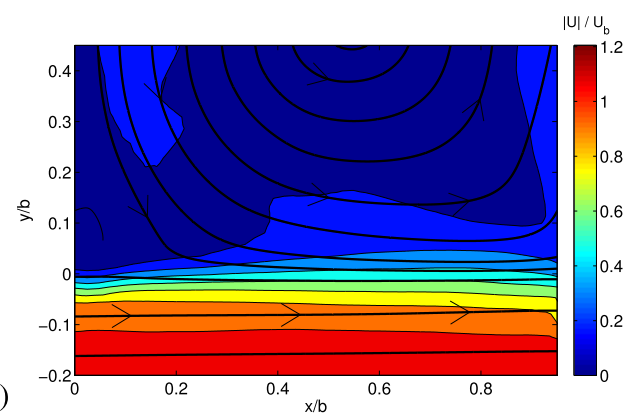

FIG. 3. (a) Approaching mean streamwise velocity profile measured at two locations in the upstream flow at the horizontal plane $z_{L}=0.05 \mathrm{~m}$; and (b) streamlines and time-averaged velocity magnitude measured at the cavity interface, at elevation $z_{L}=0.05 \mathrm{~m}$. 

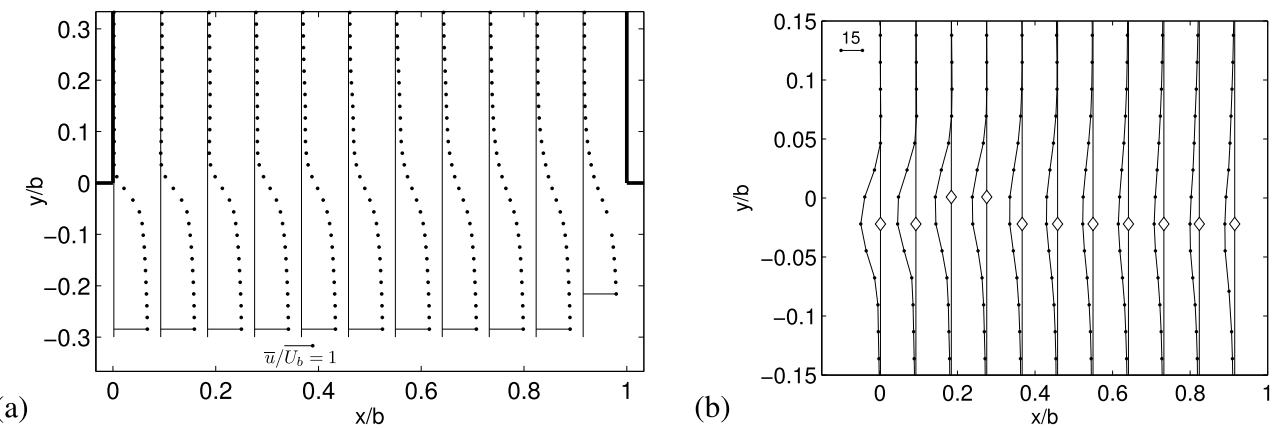

FIG. 4. (a) Transverse profiles of time-averaged non-dimensional streamwise velocity $\bar{u}$; and (b) Non-dimensional transverse gradient of mean streamwise velocity $\frac{b}{U_{b}} \frac{\partial \bar{u}}{\partial y}$ at the interface with white symbols indicating the location of maximum gradient (the streamwise spatial resolution is reduced for better reading).

entrainment that produces the lateral spreading of the mixing zone. This is clearer when we observe the transverse velocity gradient in Figure 4(b), where in this case the maximum gradient is slightly deflected towards the main stream $(y / b<0)$ in most of the interface between the cavity and the main channel.

The spreading of the mixing layer is analyzed by expressing its thickness $\delta$ as a function of the difference between characteristic velocity scales at each side of the interface. We define these velocities at each streamwise location $x$ inside the cavity $U_{1}(x)$, and in the main channel $U_{2}(x)$, as the mean streamwise velocity at the position along the $y$ axis where the absolute value of the velocity gradient becomes negligibly small, i.e., lower than $0.5 \mathrm{~s}^{-1}$. The measured mixing layer thickness $\delta_{m}(x)$ is then defined as follows:

$$
\delta_{m}(x)=\frac{U_{2}(x)-U_{1}(x)}{|\partial \bar{u}(x) / \partial y|_{\max }},
$$

where $|\partial \bar{u}(x) / \partial y|_{\max }$ is the maximum transverse gradient of the mean streamwise velocity at each position $x / b$ along the interface. As shown in Figure 5, the mixing-layer thickness not only increases in the downstream direction but also exhibits an interesting feature near the downstream corner of the interface $(x / b>0.8)$ where $\delta_{m}$ decreases due to a local increase of the maximum velocity gradient. This is a consequence of the adverse pressure gradient generated by the downstream corner of the cavity, where the shear-layer impinges the wall increasing locally the magnitude of the pressure.

To identify the sensitivity of the mixing layer to the adverse pressure gradient, we can compare the development of the mixing layer with the theoretical growth under free conditions. The thickness of a free mixing layer $\delta_{e}$, can be approximated by using the following relationship:

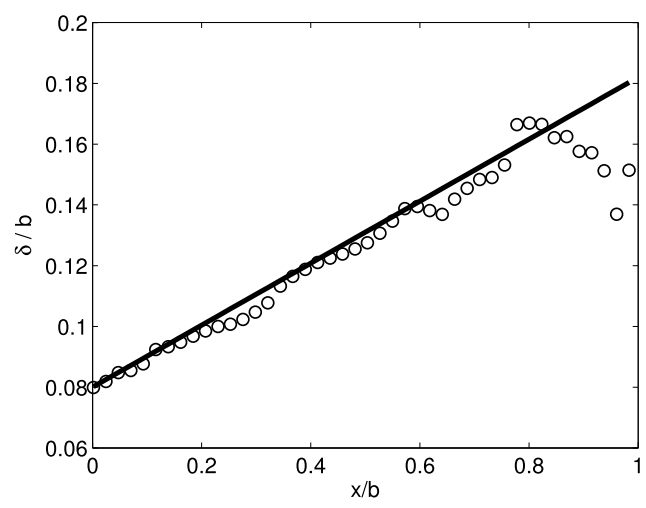

FIG. 5. Streamwise evolution of the measured $\left(\delta_{m}, \circ\right)$ and theoretical width $\left(\delta_{e}\right.$, solid line $)$ of the mixing-layer along the interface of the main channel and the cavity. 


$$
\frac{d \delta_{e}}{d x}=\alpha \frac{\widetilde{U}_{2}-\widetilde{U}_{1}}{\left(\widetilde{U}_{1}+\widetilde{U}_{2}\right) / 2},
$$

where $\widetilde{U}_{1}$ and $\widetilde{U}_{2}$ are constant velocity scales that represent the magnitudes of the velocity field inside and outside the cavity, respectively, and $\alpha$ is a constant that modulates the growth of the shear-layer in the streamwise direction. According to Pope ${ }^{22}$ the range of $\alpha$ is between 0.06 and 0.11 , while Lesieur $^{26}$ proposes a constant value of 0.085 . To compare with the measured data, we consider that the scale of the outer velocity in the main channel $\widetilde{U}_{2}$ can be represented by the bulk velocity of the flow, such that $\widetilde{U}_{2} \approx U_{b}$, and the velocity scale in the cavity is approximated by $\widetilde{U}_{1} \approx 0.15 U_{b}$, as estimated in Figure 2, with a mixing-layer growth rate coefficient of $\alpha=0.069$ that yields a best-fit slope of $\delta_{m}(x)$ equal to 0.102 , as shown in Figure 5. Assuming an initial width of $0.08 b$, it appears that the adverse pressure gradient only affects the thickness of the mixing layer for $x / b>0.8$, where it deviates from the linear growth. This is a remarkable characteristic of the flow, since the shear-layer develops in a very short and restrained section at the interface. The thickness growth preserves this property, even though the shear-layer interacts continuously with the vortices that emerge from inside the cavity, which are discussed later in the paper, and it is affected by the strong adverse pressure gradient produced at the impinging point, in the downstream corner of the cavity.

Turbulent transport of momentum across the interface can be quantified through the primary term of the Reynolds stress tensor. Figure 6 reveals that at each selected streamwise location $x$, the maximum Reynolds shear stress magnitude $\left|\overline{u^{\prime} v^{\prime}}\right|$ is located along the centerline of the mixing layer $(y / b \approx 0)$ and rapidly vanishes on both sides at a distance equal to about $\pm \delta_{m} / 2$, except near the downstream end of the mixing layer $(x / b>0.8)$ where it spreads further away as $\delta_{m}$ suddenly decreases, as already observed in Figure 5. As expected, Figure 6 also shows that the magnitude of the dimensionless maximum Reynolds shear stress increases in the downstream direction, reaching a maximum near $x / b=0.7$, and decreasing again towards the downstream corner due to the effect of the adverse pressure gradient imposed by the stagnation point. To summarize, the flow at the interface between the main stream and the cavity exhibits typical characteristics of developing free mixing layers, i.e., the maximum velocity gradient and maximum turbulent shear stress take place close to the interface and spread in the downstream direction. The most important feature of the mixing layer at the cavity interface is the sensitivity to the adverse pressure gradient that only affects the time-averaged characteristics of the shear-layer for $x / b>0.8$, decreasing the width and maximum Reynolds shear stress when approaching the downstream corner.

\section{COHERENT STRUCTURE DYNAMICS IN THE MIXING-LAYER}

The objective of the present section is to analyze the temporal characteristics of the turbulent coherent structures in the mixing layer. We first identify the leading frequency of the vortex shedding

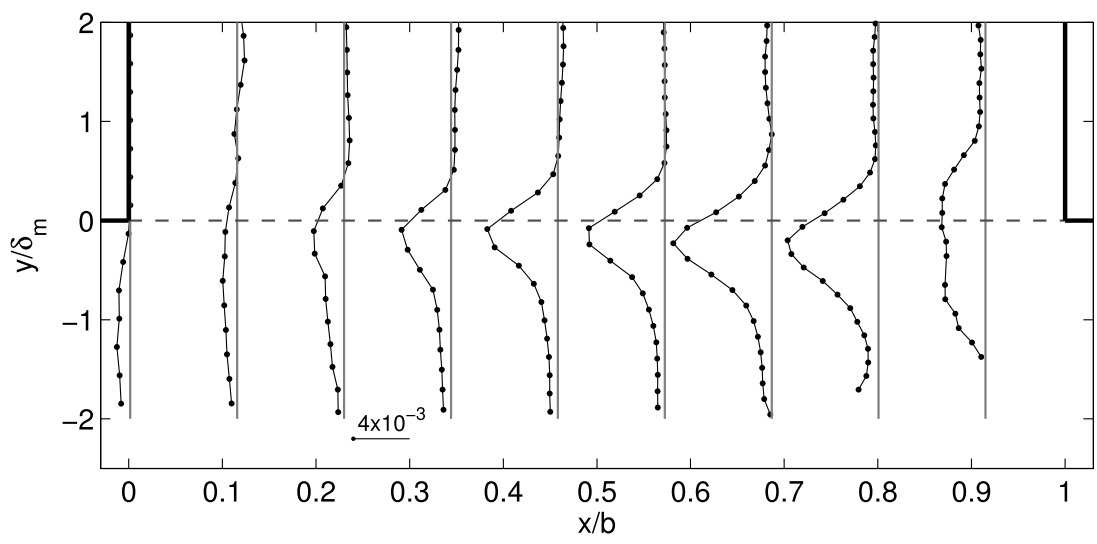

FIG. 6. Turbulent transport of momentum is quantified by the evolution of transverse profiles of the non-dimensional Reynolds shear stress $-\overline{u^{\prime} v^{\prime}} /\left(U_{2}-U_{1}\right)^{2}$ in the streamwise direction along the mixing layer. 


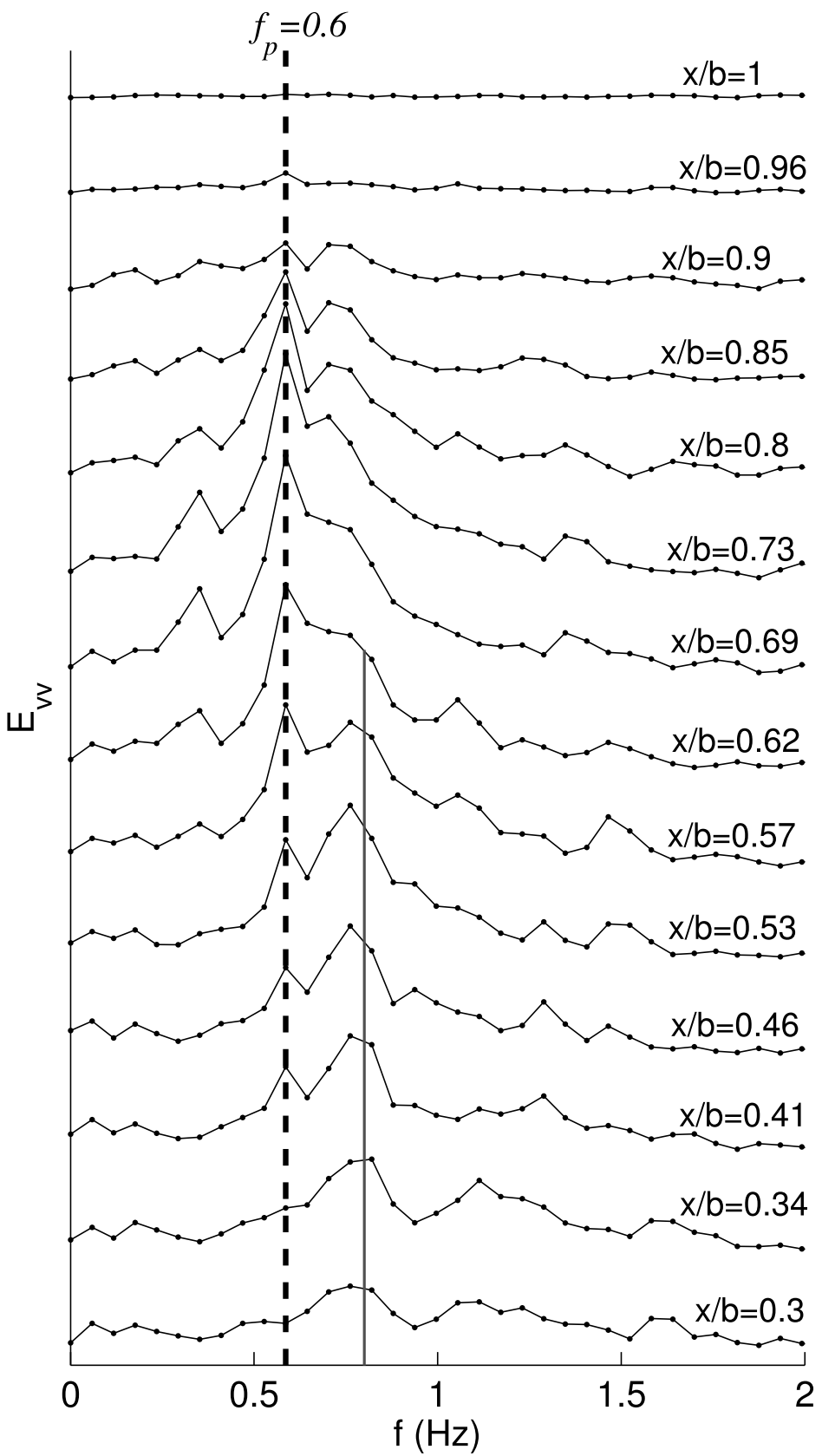

FIG. 7. Power spectral density of the transverse velocity across the interface $v$, plotted at different streamwise locations. A similar scaling is used for all spectra.

by plotting the streamwise evolution of the power spectral density of the transverse velocity signal $v$ at the interface, as shown in Figure 7, similar to what was reported by Rockwell and Naudascher. ${ }^{1}$ The dominant frequency in the downstream region of the mixing layer $(x / b>0.5)$ equals $f_{p}=0.6 \mathrm{~Hz}$, with significant energy also around $0.8 \mathrm{~Hz}$ for the first sections $(x / b<0.62)$ of the shear layer. The maximum peak of energy for $0.6 \mathrm{~Hz}$ is measured at a location $x / b=0.7$, where the transverse velocity fluctuation coherency is then maximum. These complex characteristics of the shear layer show that the turbulent flow past a lateral cavity is highly three-dimensional. The controlling mechanisms of the shear-layer dynamics, however, which are part of the analysis carried out in this section, show that the most energetic large-scale coherent structures of the flow are mostly 2D, as discussed by Tuna et al. ${ }^{9}$ 


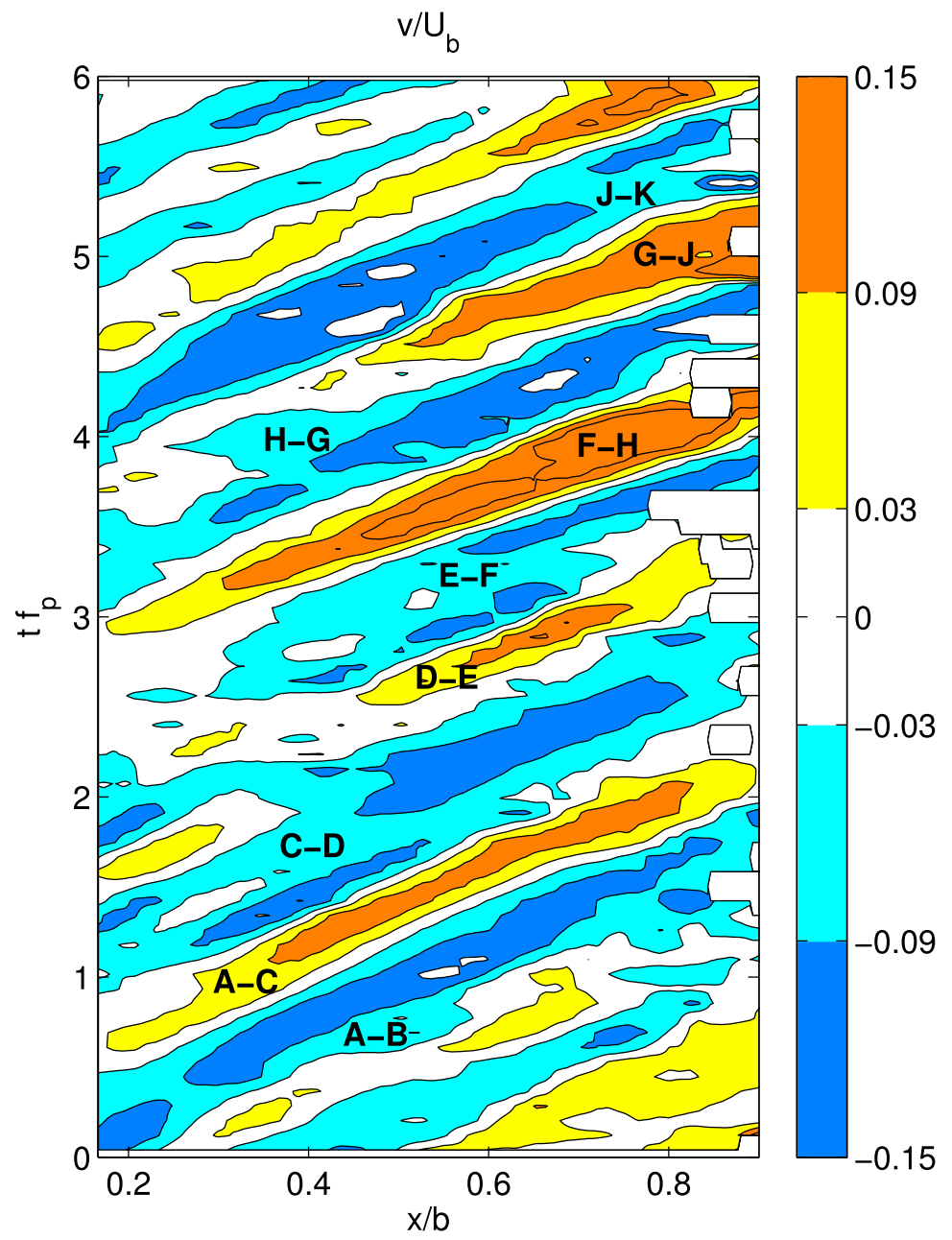

FIG. 8. Streamwise and time evolution of the non-dimensional transverse velocity component along the mixing layer. The capital letters refer to the structures from Figure 10.

In Subsections IV A-IV C we focus our attention on the 2D propagation of the vortices of the mixing layer along the interface. First we discuss the characteristics of the transverse velocity across the interface, and then we study the evolution of the low-frequency, large-scale coherent structures that are continuously shed between the cavity and main stream, by analyzing the vortex cores using the identification algorithm of Graftieaux et al. ${ }^{27}$

\section{A. Transverse velocity alternation}

The propagation of vortices generated at the interface produces an alternate sequence of positive and negative fluctuations of transverse velocity component $v$ between the cavity and the main stream. This oscillating motion is shown in Figure 8 as an alternation of streamwise and temporal contours of positive and negative transverse velocity $v$ across the interface. Through this space-time plot we can characterize the vortex evolution, illustrating efficiently important physical features of the mixing layer. The data are averaged over $0.133 \mathrm{~s}$, which corresponds to over 4 consecutive measured values for the first $10 \mathrm{~s}$ of data. Positive regions of $v$ that are indicated in yellow/orange color correspond to the flow directed towards the cavity, whereas the regions of instantaneous negative $v$ in blue correspond to the flow directed towards the main stream. It is important to note that the time-averaged velocity magnitude across the interface $\bar{v}$ is negligibly small, which indicates that the development 
of the vortices produces a symmetric sequence of positive and negative large-scale fluctuations of the same magnitude that compensate each other, yielding a near zero average.

As observed in the evolution of the spectra previously shown in Figure 7, the shear layer organizes as the vortices travel in the downstream direction, which is clearly distinguished in the range $0.3 \leq x / b \leq 0.9$. The maximum transverse velocity magnitude also increases in the downstream direction, reaching a maximum of $0.15 U_{b}$ at $x / b=0.7-0.8$ and then decreases as the shear layer decelerates due to the adverse pressure gradient. The streamwise location of this maxima is in agreement with the periodicity shown in the maximum amplitude of the spectrum observed for $x / b=0.7$, in Figure 7.

From the slope of the centerline of the alternating areas of positive and negative $v$ contours in Figure 8 , the propagation speed $c$ of the large-scale vortices at the interface can also be estimated, as they move downstream. These parallel centerlines yield a constant value of the propagation speed, equal to $c=0.094 \mathrm{~m} / \mathrm{s}=0.56 U_{b}$. The vortex propagation speed thus exceeds the mean streamwise velocity averaged along the interface. This velocity is denoted as $U_{i}$, and it is equal to $U=0.43 U_{b}$, such that $c / U_{i} \approx 1.3$. This is similar to the observations of Shaw et al. ${ }^{28}$ who showed that the coherent structures in the shear layer formed at the crest of a vegetation canopy are advected at a propagation speed equal to 2 times the mean local velocity, while Zhang et al. ${ }^{29}$ estimated this ratio as 1.6 , in better agreement with the present results. Moreover, the spacing between two consecutive high peaks (or low peaks) of transverse velocity contours correspond to the wavelength $\lambda$ of the vortices along $x$ axis, which in this case is equal to $\lambda=c / f_{p}=0.16 \mathrm{~m}=0.52 b$.

To further our understanding on the velocity fluctuations induced by the vortex shedding of the mixing layer, we plot in Figure 9 time series of velocities $u^{\prime}$ and $v^{\prime}$, at a specific point along the interface, corresponding to $y / b=0$ and $x / b=0.7$, which is the location of maximum energy of the frequency of $0.6 \mathrm{~Hz}$ along the interface, i.e., the maximum peak of the spectrum in Figure 7. The plot in Figure 9 shows that in most cases the periodic time series of the streamwise fluctuation and the zero-mean transverse velocity are in phase, i.e., both fluctuation velocity components $u^{\prime}$ and $v^{\prime}$ are mostly of the same sign, even though the oscillation of $u^{\prime}$ appears to be more complex, as will be deeply discussed in Subsection IV B, along with the data presented in Figure 10. In the following figures we non-dimensionalize time using the leading frequency of the mixing layer, expressing the dimensionless time as $t f_{p}$.
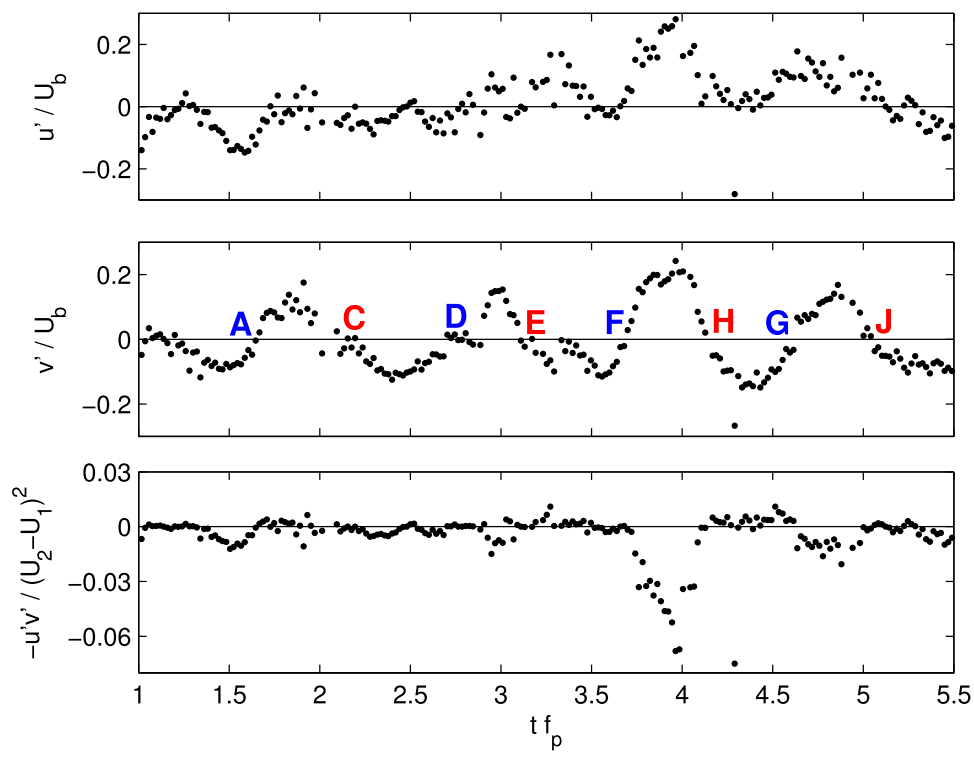

FIG. 9. Time evolution of the non-dimensional horizontal velocity fluctuations $u^{\prime}$ and $v^{\prime}$, and of the correlation $-u^{\prime} v^{\prime}$ values, at the point $y / b=0$ and $x / b=0.7$. The capital letters refer to the structures from Figure 10. Time is non-dimensionalized using the leading frequency of the mixing layer $f_{p}$. 

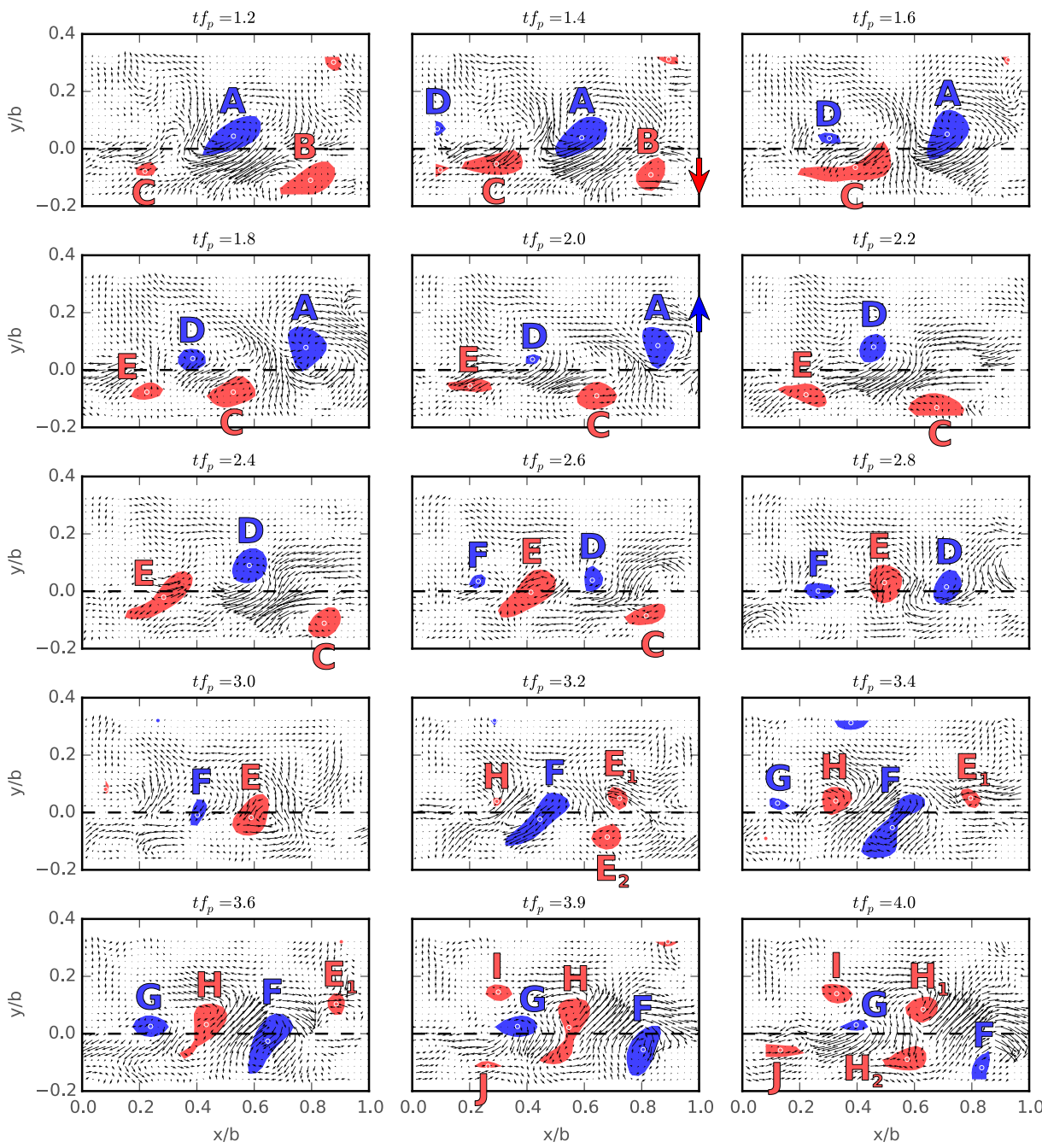

$0.2 \mathrm{Ub}_{\mathrm{b}}$

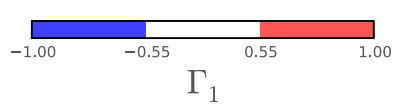

FIG. 10. Time evolution every $t f_{p}=0.2(0.33 \mathrm{~s})$ of the fluctuating velocity field (back arrows) and contours of clockwise (blue color, $\Gamma_{1}<-0.55$ ) and counter-clockwise (red color, $\Gamma_{1}>0.55$ ) coherent vortices, where the white circles correspond to the vortex centers, i.e., the maximum local value of $\left|\Gamma_{1}\right|$. The dashed line represents the mixing interface, $y / b=0$.

A quadrant analysis performed at the same point (not shown herein) confirms that the values of the velocity fluctuations are both positive, $v^{\prime}>0, u^{\prime}>0$, during $33 \%$ of the time, locating the measurements in the first quadrant. Similarly, both velocity fluctuations are negative, $v^{\prime}<0, u^{\prime}<0$, positioning the measurements in the third quadrant $40 \%$ of the total measurement time. These conditions lead to a mostly negative instantaneous turbulent stresses, as shown in the lower subplot of Figure 9, confirming also the negative time-averaged Reynolds shear stress $-\overline{u^{\prime} v^{\prime}}$ depicted in Figure 6 . The alternation of the transverse velocity fluctuations, which include intense positive and negative transverse velocity events, is likely responsible for the exchange of passive scalars and particulate matter between the main stream and the cavity that has been observed in experimental studies of surface storage zones.

\section{B. Two-dimensional time evolution of vortices in the plane}

The study of the time series performed in Subsection IV A showed specific features of the mixing layer along the interface, which are a consequence of the vortex shedding. In this section we carry out 
a spatial analysis, considering the entire measurement plane, to reveal the vortex related mechanisms that produce the large-scale oscillations of streamwise and transverse velocities. We follow the methodology proposed by Graftieaux et al. ${ }^{27}$ to extract the center of low-frequency, large-scale coherent structures that are continuously shed and advected downstream. This vortex identification method first employs the proper orthogonal decomposition (POD) ${ }^{30}$ to extract the most energetic modes of the flow through a linear decomposition. It then defines a non-dimensional scalar function, denoted as $\Gamma_{1}$, that is used to identify the center of the vortices based on the orientation of the velocity field. In our analysis of the shear layer, we carry out the vortex identification procedure in the following three steps:

1. We first perform a POD of the two-dimensional field of velocity fluctuations (after subtracting the mean flow) over a total time of $133 \mathrm{~s}$ with a sampling frequency of $30 \mathrm{~Hz}$ which permits to follow the trajectories of the structures as shown in Figure 10. We then extract the eigenmodes and use the 10 largest modes to filter and reconstruct the velocity fields, keeping $90 \%$ of the mean kinetic energy and removing a large part of the non-coherent turbulent kinetic energy (about $60 \%$ ) and of the measurement noise. For more details on the basic aspects of the POD theory, the reader is referred to Holmes et al. ${ }^{30}$

2. Subsequently, we calculate at each time step the spatial distribution of the dimensionless scalar function $\Gamma_{1}$, using the reconstructed velocity field. This function can be written for any point $P$ of the domain as follows:

$$
\Gamma_{1}(P)=\frac{1}{S} \int_{M \in S} \frac{(\overrightarrow{P M} \times \vec{U}(M)) \cdot \vec{z}}{\|\overrightarrow{P M}\| \cdot\|\vec{U}(M)\|} d S=\frac{1}{S} \int_{M \in S} \sin \left(\theta_{M}\right) d S,
$$

where $S$ a circular surface centered on $P, M$ a point within $S, \vec{U}(M)$ the velocity vector at point $M, z$ the previously defined vertical axis, and finally $\theta_{M}$ the angle between vectors $\overrightarrow{P M}$ and $\vec{U}(M)$. As the sine function is bounded by 1 and the integral is normalized by the integration area $S,\left|\Gamma_{1}\right|$ is also bounded by 1 . In order to capture the turbulent structures, the integration area has to be of same size as the typical extension of the structures: in the present case, we selected a circle of diameter equal to $60 \mathrm{~mm}(0.2 \mathrm{~b})$, which is the order of magnitude of the size of the coherent structures in the fluctuating velocity field (black arrows) in Figures 10 and 12.

3. We finally select a threshold value of $\Gamma_{1}$ to visualize the vortex cores in regions of high rotation. In this study we choose $\left|\Gamma_{1}\right|=0.55$ as it appears to be adapted for the identification of the coherent vortices in Figure 10. Contours of counter-clockwise and clockwise vortices are plotted on the following figures, with the red color for counter-clockwise with $\Gamma_{1}>0.55$, and blue for clockwise with $\Gamma_{1}<-0.55$.

Note that this method was selected rather than a method based on a threshold of vorticity as (i) unlike the vorticity, the magnitude of $\Gamma_{1}$ remains limited in purely shear flow regions and (ii) it allows us to follow each vortex along most of the mixing layer, from its upstream region where they are weakly energetic, to its downstream region where they become highly energetic.

In Figure 10 we present a sequence of the vortex dynamics at 15 consecutive times, advancing from the upper left plot to the lower right, and visualized every $0.33 \mathrm{~s}$ for a total time of $5.0 \mathrm{~s}$. The time in Figure 10 is non-dimensionalized by the leading frequency of the shear-layer $f_{p}$, such that the dynamics of the vortices can be observed in the context of the fundamental period of the vortex shedding. In each plot we show the instantaneous (not-filtered) fluctuating velocity field with black arrows, which is averaged over 4 consecutive measurements, reducing the sampling frequency from $30 \mathrm{~Hz}$ to $7.5 \mathrm{~Hz}$. The vortex cores are identified with the $\Gamma_{1}$ criterion, ${ }^{27}$ and they are depicted in blue or red colors, depending if the rotation is clockwise or counter-clockwise, respectively. To analyze the evolution in time of the coherent structures, the vortices are individually identified by a letter to observe their trajectory, as they are advected along the mixing layer.

The evolution of the coherent structures visualized in Figure 10 shows that the instantaneous flow is highly dynamic and characterized by the formation and interaction of multiple vortices. In the entire plane we can distinguish from two to six vortical structures, which are seen to interact and 
sometimes split. It is important to point out that the instants observed in the $2 \mathrm{D}$ plots of Figure 10 coincide with the time in the velocity series at $x / b=0.7$ and $y / b=0$ depicted in Figure 9, which can be used to compare the global vortex interaction with the velocity components measured at one point.

From the series of snapshots in Figure 10 that are representative of the dynamical processes in the mixing layer, it appears that three main configurations control the flow at the interface: (1) From $t f_{p}=1.2$ to $t f_{p}=2.6$, the clockwise vortices (blue) are located inside the cavity $(y / b>0)$, whereas the counter-clockwise vortices (red) reside in the main stream $(y / b<0)$. In this configuration, two intense transverse velocity regions occur along the interface $(y / b=0)$, the first is upstream from the clockwise vortices, where $v^{\prime}>0$ and $u^{\prime} \approx 0$ (as seen upstream of vortex $A$ at $x / b=0.7$ and $t f_{p}=1.8-2.0$, see also Figure 9), and the second is downstream from the clockwise vortices, where $v^{\prime}<0$ and $u^{\prime}<0$ (as seen downstream of vortex $A$ at $x / b=0.7$, and $t f_{p}=1.4-1.6$, see also Figure 9). (2) From $t f_{p}=2.8$ to $t f_{p}=3.4$ the vortices are aligned with the interface at $y / b \approx 0$. In this configuration, $v^{\prime}$ still oscillates between positive and negative values, but the streamwise component $u^{\prime}$ exhibits a more complex pattern (see $x / b=0.7$ at times $t f_{p}=2.8-3.4$, see also Figure 9). (3) Finally, from $t f_{p}=3.6$ to $t f_{p}=4.0$, the clockwise vortices (blue) are located in the main stream $(y / b<0)$, and the counter-clockwise vortices (red) are located inside the cavity $(y / b>0)$. The velocity fluctuations along the interface exhibit an opposite behavior compared to configuration (1): upstream from the clockwise vortices the fluctuations of velocity are both positive, $v^{\prime}>0$ and $u^{\prime}>0$ (as seen upstream of vortex $F$ at $x / b=0.7$ and $t f_{p}=3.9-4.0$, see also Figure 9); and negative transverse velocities are observed downstream from the clockwise vortices, $v^{\prime}<0$ and $u^{\prime} \approx 0$ (as seen downstream from vortex $F$ at $x / b=0.7$ and $t f_{p}=3.6$, see also Figure 9).

Additional aspects of the flow are revealed from the analysis of the vortex-core evolution. Processes such as the alignment of vortices at the interface and the vortex splitting produce more complex fluctuations of the streamwise velocity compared to the transverse component, as previously observed in the time series of Figure 9. As also seen in our analysis, both components of the fluctuating velocity field have the same sign most of the time. This positive correlation between $u^{\prime}$ and $v^{\prime}$ generates negative Reynolds stresses, and it is mostly produced when the absolute value of the streamwise component $\left|u^{\prime}\right|$ is intense. In these cases two dominant motions are observed, either $u^{\prime}$ and $v^{\prime}$ positive, which corresponds to the third vortex configuration discussed in the previous paragraph, or $u^{\prime}$ and $v^{\prime}$ are both negative which corresponds to the first configuration. Both cases had been recently reported by Sanjou and Nezu, ${ }^{11}$ who extended the classical terminology "ejection" and "sweep" to an open-channel lateral cavity flow.

As previously noted, some of the coherent structures go through a splitting process, creating two separate co-rotating cores at each side of the interface that emerge from one original structure of the shear layer. This is observed for vortex $E$ at $t f_{p}=3.2$, and vortex $H$ at $t f_{p}=3.9$ in Figure 10 . The leading edge of the co-rotating vortex pair that travels downstream produces the largest velocity fluctuations at the interface, as revealed by the time series of velocity depicted in Figure 9.

As the coherent vortices reach the downstream corner, where the shear-layer impinges the wall $(x / b=1)$, they either enter the cavity $(y / b>0)$ or continue to the main stream $(y / b<0)$. By defining the vortex location as the center of gravity of the colored area given by $\left|\Gamma_{1}\right|>0.55$, indicated by blue open circles in Figure 11, we can observe that the vortex center path depends mostly on the location of this center with respect to the interface, upstream from the impinging point. For instance, vortices $A$ and $E 1$, which are located at $y / b>0$, are deflected toward the cavity, whereas vortices $B, C, E 2$, and $F$, which are located at $y / b \leq 0$, continue downstream in the main channel.

Figure 11 shows the time-space evolution of the vortex center locations during the same period of $10 \mathrm{~s}$ as in previous figures. We plot the streamwise location along the $x$ axis of all the vortex cores encountered near the interface, in the region $|y / b|<0.167$. The coherent structures shown in Figure 11 correspond to the same vortices identified in our previous analysis, which were defined by the letters $A$ to $J$ in Figure 10. The time-evolutions of these streamwise locations exhibit an approximately constant slope in the time-space plot, confirming the constant streamwise propagation speed of the coherent structures of the mixing layer, reported in Subsection IV A. The period defined as the characteristic time scale between two structures with the same rotation sign, passing through the same streamwise location along the $x$ axis is equal to $t f_{p}=1.0$. 


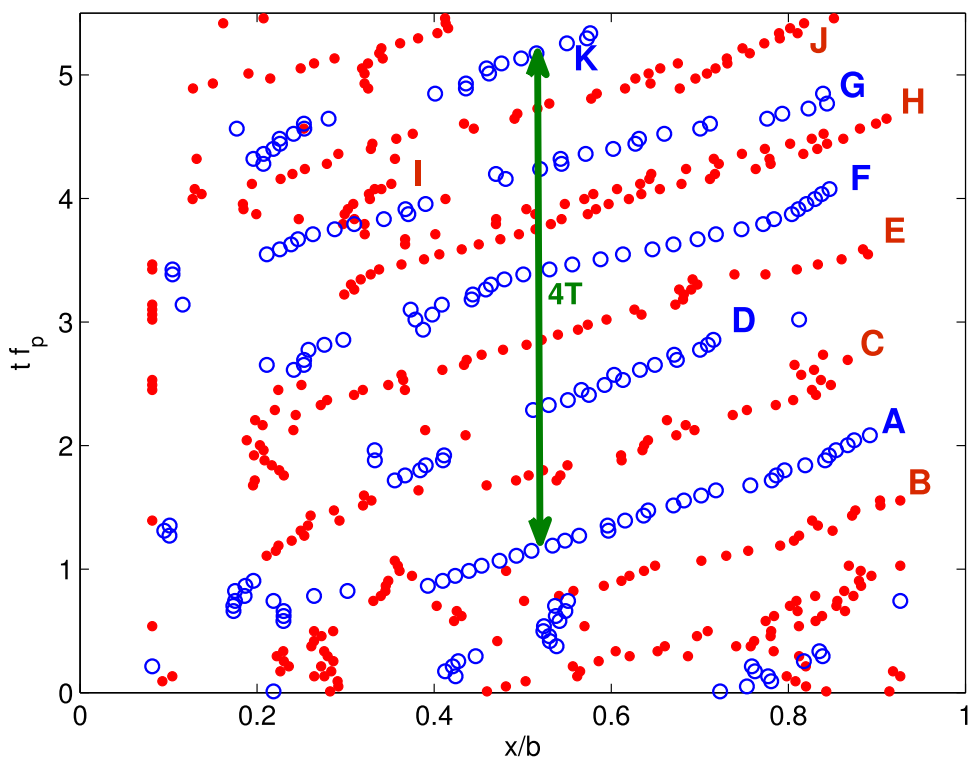

FIG. 11. Time-evolution of the streamwise location of the clockwise (open blue symbols) and counter-clockwise (closed red symbols) vortex centers located near the interface (with $|y / b|<0.167$ ). Green arrow designates four periods.

\section{Interaction with inner vortices and statistics of core locations}

The vortices described above emerge near the upstream corner and are advected along the interface of the main channel with the cavity. These structures, such as the vortices identified with the letters $A$ to $J$ in previous figures, are originated near the leading edge of the cavity $x / b=0$ and move downstream with an approximately constant vortex propagation speed. The analysis of the vortex cores in the vicinity of the interface, however, shows that additional vortical structures that are generated inside the cavity can interact with the coherent structures of the mixing layer. In Figure 12 we plot the instantaneous locations of vortex cores using the $\Gamma_{1}$ criterion, during short episodes in which these vortices are strong enough to reach the interface and interact with the shear-layer structures. These inner vortices, identified as $L$ (clockwise) and $M$ (counter-clockwise) in Figure 12, emerge directly within the cavity. The process responsible for their appearance remains unclear; one hypothesis is that they emerge from the smaller shear-layer produced between the recirculating region at the corner, and the large-scale recirculating cell that occupies most of the cavity (see Figure 2).

Nevertheless, the dynamics of the flow measured in our experiments shows that the formation of these vortices and their interaction with the mixing layer at the interface is very sporadic. During the total measurement time, only $3 \%$ of the coherent structures identified in the analysis correspond to inner vortices from the cavity. As they approach the interface, the higher velocities of the main channel stretch these structures in an oblique direction, rapidly moving in the positive $x$ and negative $y$ directions. In this situation, the inner vortices also undergo a splitting process, separating into two smaller structures, one ( $L 1$ and $M 1$ ) staying inside the cavity, and the second one ( $L 2$ and $M 2$ ) being advected toward the mixing layer as shown in Figure 12.

With the objective of demonstrating the statistical significance of the processes we have unveiled, and that the leading frequency of the shear-layer is the fundamental mechanism of the flow at the interface, we show in Figure 13 more details on the POD analysis applied to the transverse velocity, in the same region depicted in Figures 10 and 12. Figure 13(a) shows the POD spectrum, where the two largest modes represent a significant percentage of the transverse velocity variance. The frequency spectra of these two modes, depicted in Figure 13(b), show that both largest modes have the same peak, at the leading frequency of the shear-layer $f_{p}=0.6 \mathrm{~Hz}$. Figures 13(c) and 13(d) show these two spatial modes, with the large structures that comprise the mixing layer in the decomposition. Higher-order modes (not shown herein) exhibit from 3 to 5 vortical structures that form the fundamental dynamics of the shear-layer. From the third to the sixth modes, the POD captures the inner 

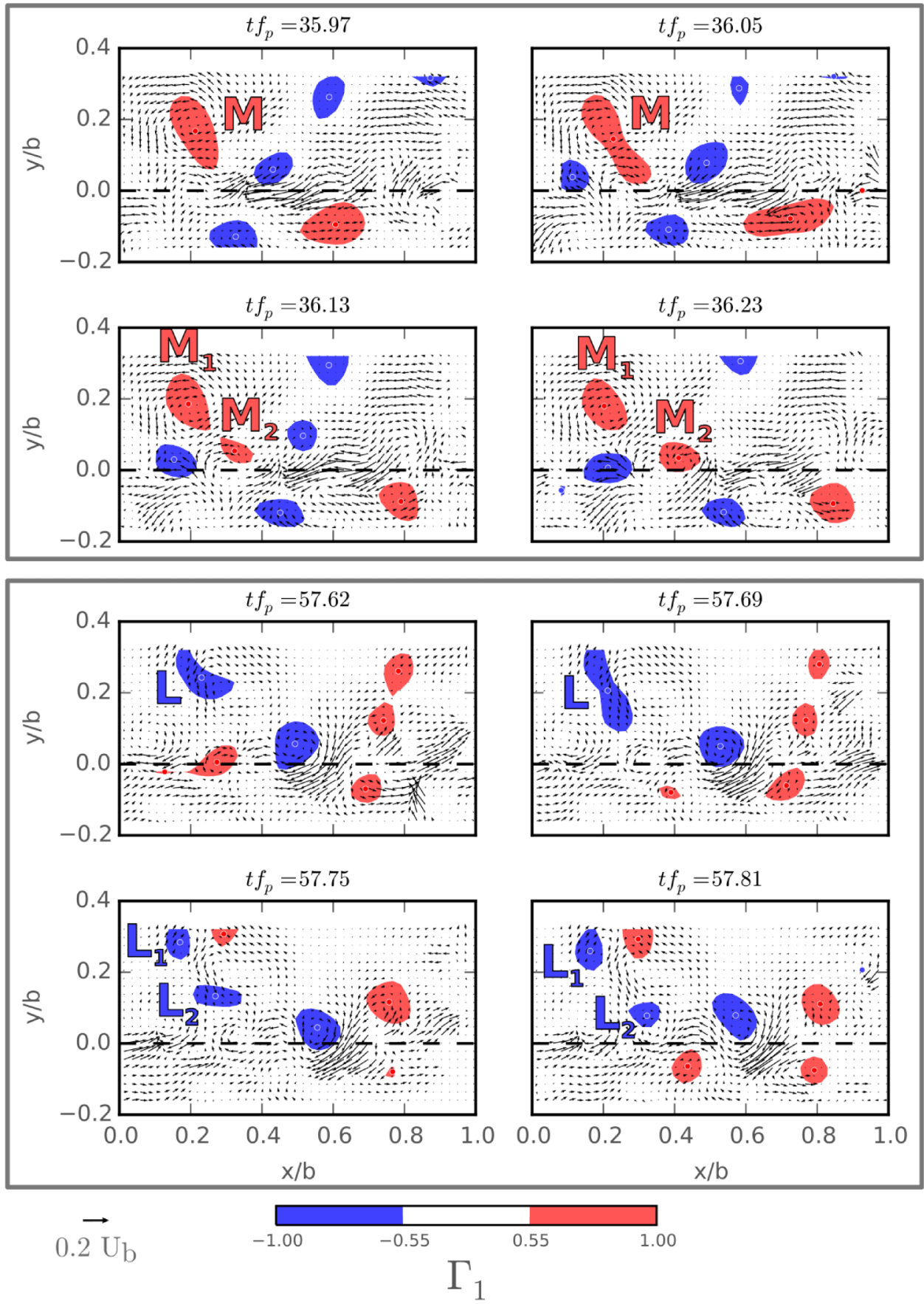

FIG. 12. Time evolution of the fluctuating velocity field (black arrows) and contours of clockwise (blue color, $\Gamma_{1}<-0.55$ ) and counter-clockwise (red color, $\Gamma_{1}>0.55$ ) coherent vortices, to capture the dynamics of inner vortices $L$ and $M$ that originate inside the cavity and interact with the mixing layer. The dashed line represents the mixing interface, $y / b=0$.

vortices that interact with the shear layer in the first section of the interface, which were previously discussed in the analysis of Figure 12.

In Subsections IV A and IV B, we have provided a description of the complex vortex dynamics and interactions that occur in the shear-layer. To visualize the spatial extension occupied by these large-scale vortices, we plot in Figure 14 the probability density function (pdf) of the vortex core locations in the vicinity of the interface: the integral of this estimate over a small area within the 

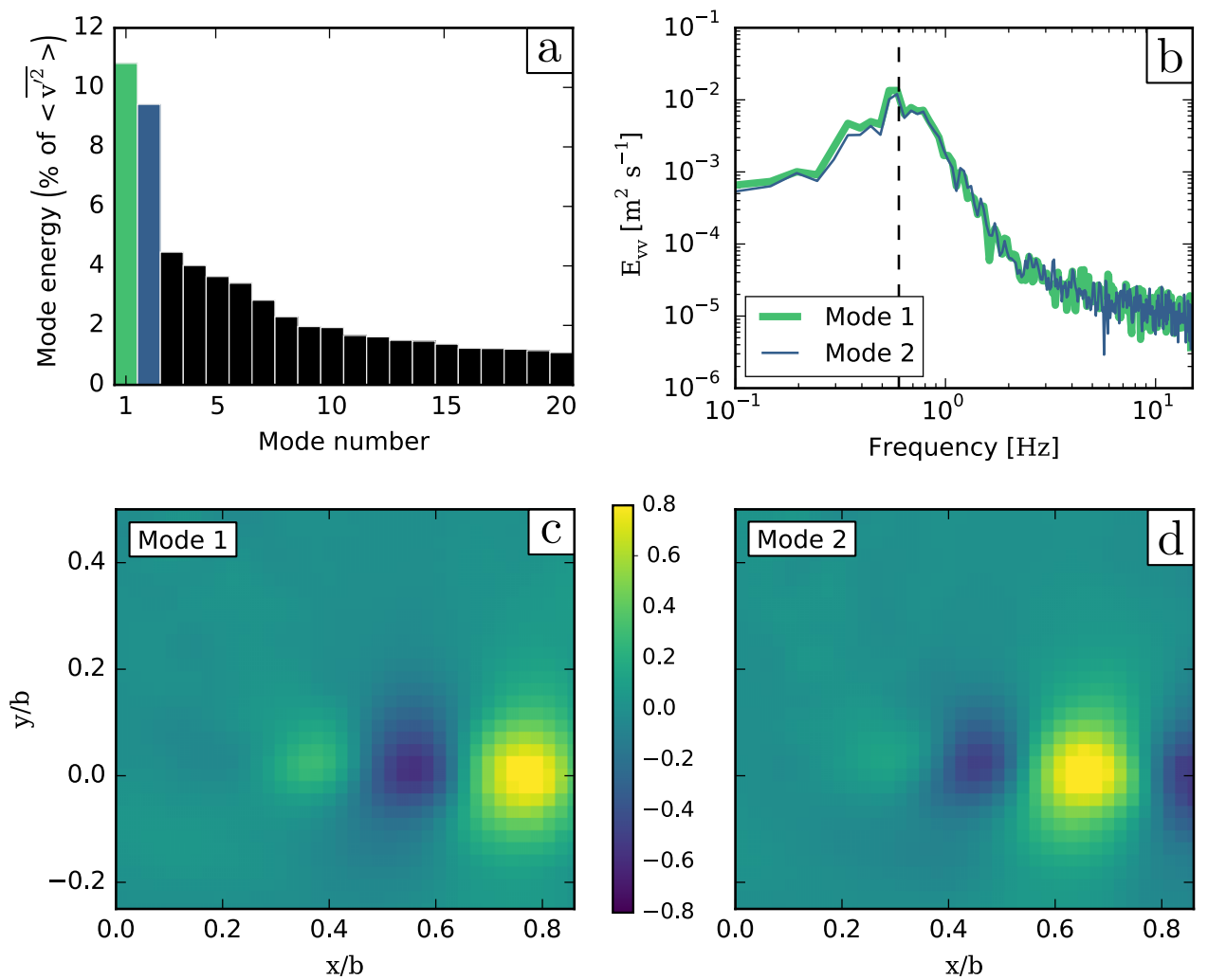

FIG. 13. Proper orthogonal decomposition (POD) of the transverse velocity fluctuations in the vicinity of the interface. (a) POD spectrum shows that two largest modes represent a significant percentage of the transverse velocity variance; (b) Frequency spectrum of the coefficients of the first two modes reveals that both have a peak at $f_{p}=0.6 \mathrm{~Hz}$ (dashed vertical line); (c) Spatial component of mode 1; and (d) Spatial component of mode 2.

whole domain is equal to the probability that a vortex core is located within this area. ${ }^{31}$ The $2 \mathrm{D}$ plots show contours of normalized histograms, such that the sum of the integrals of the two pdfs, clockwise and counter-clockwise vortex cores, respectively, on the plotted domains $(0.1<x / b<0.9$ and $-0.15<y / b<0.25)$ is equal to 1 .

The pdfs shown in Figure 14 reveal that the locations of clockwise and counter-clockwise rotating vortices have almost the same statistical spatial distribution. In the upstream region $(x / b<0.3)$, we can observe the high density of inner vortices $(y / b \approx 0.15)$, such as $L$ and $M$ in Figure 12, and that of the vortex cores originating in the shear-layer slightly inside the cavity. Further downstream $(0.3<x / b<0.7)$, vortices are predominantly located along the interface, slightly deflected towards the lateral cavity, $y / b>0$. In the downstream region $(x / b>0.7)$, two tongues of high density of vortex locations can be observed, since the structures either enter the cavity $(y / b>0)$ or continue to the main stream $(y / b<0)$ as the shear-layer impinges on the corner. These two distinct regions of higher

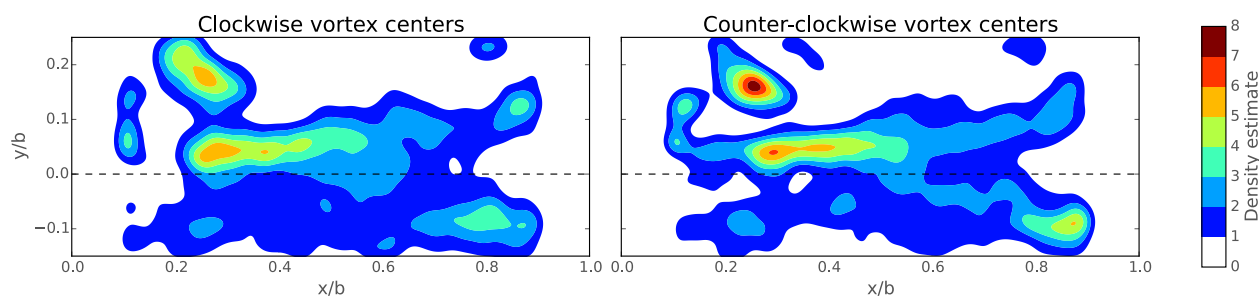

FIG. 14. Probability density functions (pdfs) of vortex center locations identified using the $\Gamma_{1}$ criterion in the vicinity of the interface. (a) Clockwise vortex locations pdf; (b) counter-clockwise vortex locations pdf. Note that 6632 clockwise and 6423 counter-clockwise detected vortices were obtained over the 3000 samples and used to create the figure. 
densities in Figure 14 comprise the area given by $-0.1<y / b<0.1$ and appear from $x / b>0.8$, which corresponds to the location where the mixing-layer shows a change in its dynamics compared to free shear-layers, as discussed in Secs. III and IV.

\section{MASS EXCHANGE COEFFICIENT}

The large-scale vortices that are continuously shed from the mixing-layer and advected along the interface, produce a periodic alternation of positive and negative fluctuations of transverse velocity, as shown in Figure 9(b). Large-magnitude events of these fluctuations, which reach up to $0.2 U_{b}$, are the most important mechanism of mass and momentum exchange between the lateral cavity and the main flow. In this section we quantify the mass exchange by computing the coefficient used in the first-order 1D model ${ }^{4}$ that represents the mass transfer of a passive scalar across the interface.

The 1D mass exchange model between the main channel and the surface storage zone, first proposed by Valentine and Wood ${ }^{32}$ and Uijttewaal et al., ${ }^{12}$ establishes a first-order relation of the mass exchange, assuming that the total mass in the cavity evolves in time as a function of the geometry, the concentration gradient, and the velocity at the interface. This relation is therefore written as follows:

$$
\frac{d M}{d t}=S_{e x} V_{e x} \Delta C(t)
$$

where $M$ is the total mass of the passive scalar within the cavity at time $t, S_{e x}=b h$ is the area of the interface section between the main stream and the cavity (see Figure 1), $V_{e x}$ is the velocity scale for the mass exchange, which is the typical time and space averaged exchange velocity across the interface, and $\Delta C$ is the passive scalar concentration gradient across the interface. Valentine and Wood ${ }^{32}$ proposed to relate $V_{e x}$ to the bulk velocity of the main stream as $V_{e x}=k U_{b}$, where $k$ is a constant and corresponds to the so-called non-dimensional mass exchange coefficient. Weitbrecht et al. ${ }^{14}$ and later Tuna et al. ${ }^{9}$ evaluated $V_{e x}$ based on the measurements of the transverse velocity along the interface of the main stream and the lateral cavity, integrating the absolute value of the average as follows:

$$
V_{e x}=\frac{1}{2 S_{e x}} \int_{S_{e x}} \overline{|v(x, z)|} d S,
$$

where the overbar stands for time averaging of the transverse velocity. It is worth noting that in the calculation of the velocity $V_{e x}$, we divide by 2 in Equation (5), due to the fact that the absolute value of the transverse velocity component considers the exchanges from the main stream to the cavity and vice versa, which is actually twice of the total mass exchange referred to in Equation (4). Assuming that the flow field is mostly 2D, Equation (5) for the mass exchange coefficient is written as

$$
k=\frac{1}{2 U_{b}} \int_{x / b=0}^{x / b=1} \overline{\left|v\left(x, z=z_{L}\right)\right|} d x .
$$

In Figure 15, we plot the streamwise evolution, from the upstream to the downstream corner of the cavity, of the mean absolute transverse velocity component, non-dimensionalized such that the

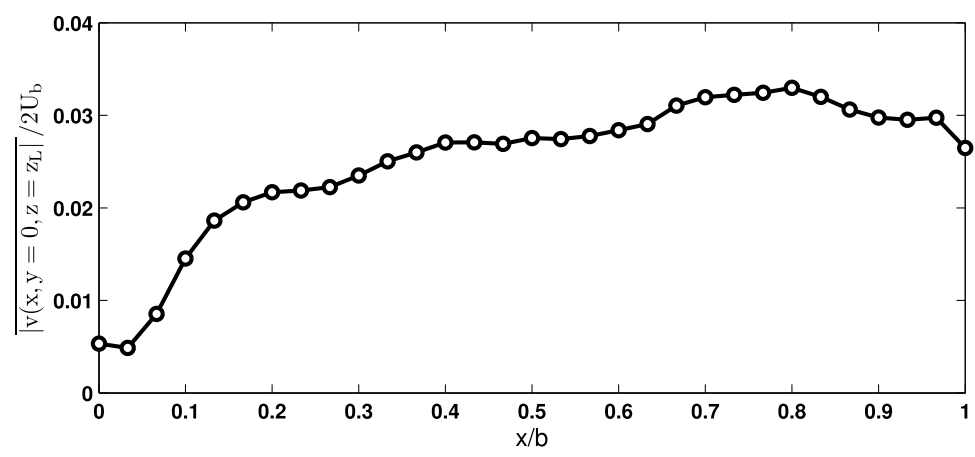

FIG. 15. Streamwise evolution of the mean absolute transverse velocity component along the interface between the cavity and the mean flow. 
averaged value between $x / b=0$ and 1 of the curve is equal to $k$. The variable plotted in Figure 15 is then directly related to the amplitude of the alternating transverse velocity component in Figure 9(b). The curve appears to increase from the upstream corner towards downstream, reach a maximum magnitude at $x / b \approx 0.7-0.8$, and to decrease again toward the downstream corner of the cavity. The maximum absolute value of transverse velocity near $x / b \approx 0.7-0.8$ is in good agreement with the location of the spectrum with a maximum value for $f_{p}$ (Figure 7), and transverse velocity magnitude (Figure 8) measured at this location in Sec. IV.

In the present case, the mass exchange coefficients yields a value of $k=0.0252$, which is in the range of the values described in the literature in cavity flows without seiching, (see, for instance, the magnitude of $k$ recently obtained by Tuna et al. ${ }^{9}$ and by Sanjou and Nezu, ${ }^{11}$ both for different aspect ratios of the cavity and mean flow characteristics). Further analyses will require to compare the value of the exchange coefficient with the transport of a passive scalar past the cavity. In this case, the complement with high-resolution numerical simulations can improve the description of the mass exchange at the interface.

\section{CONCLUSIONS}

In this investigation we analyze the experimental flow fields measured with PIV in a horizontal plane across the mixing layer that develops at the interface between a straight rectangular openchannel, and an adjacent lateral square cavity. The flow is mainly characterized by the periodic dynamics of the self-sustained shear or mixing layer that develops at the interface. The vortices advected along the mixing layer impinge on the downstream corner of the cavity, where the shear-layer is divided with some vortices entering the recirculating region in the cavity, and others continuing in the main stream downstream.

The development of the mean velocity profiles and velocity gradients at the interface, as well as the growth of the mixing layer thickness, exhibit the same characteristics as free shear-layers reported in the literature. The dynamics of the vortices, however, changes considerably from $x / b>0.8$ due to the adverse pressure gradient, as the vortices of the shear-layer approach the downstream corner of the cavity.

Measurements of the flow field have shown that along the interface the transverse velocity exhibits a zero-mean periodic signal, while the streamwise component is more complex and strongly affected by the passing of the vortices in time. To investigate further the dynamics of these coherent structures we performed a 2D analysis of their motion by combining POD with a vortex identification technique, ${ }^{27}$ employing a scalar calculated from the reconstructed velocity field, denoted as $\Gamma_{1}$. Through this analysis we demonstrated that the vortices of the shear-layer move with a constant vortex propagation speed. We also identified the splitting of vortices into two co-rotating structures as they approach the impinging point. Secondary effects are produced by the interaction of the mixing layer with vortices that are generated in a smaller shear-layer inside the cavity. Additionally, through the POD analysis we show that the periodic alternation of counter-rotating vortices is responsible for the large transverse velocity fluctuations produced at the interface by the leading edge of vortices. The dynamics of this fluctuating transverse velocity finally allowed us to estimate a 1D mass exchange coefficient across the cavity/flow interface.

In future work we aim at combining additional experiments with high-resolution numerical simulations to understand the changes on the mixing layer and flow field inside the cavity, as a function of the parameters that characterize the flow, i.e., approaching boundary layer thickness, normalized water depth, Reynolds number, and aspect ratio of the cavity. We will study the potential influences on the flow of the free surface, and waves that can be formed in the cavity. We will also focus on the three-dimensional interactions of the vortices and their global effects on mass transport to understand the mechanisms that can control the fate of contaminants in rivers.

\section{ACKNOWLEDGMENTS}

This work was supported by ECOS-Conicyt No. C11E02 and Fondecyt No. 1130940 grants. We also acknowledge the funding from Conicyt/Fondap Grant No. 15110017 and the PhD grant of W. Cai from the Chinese Scholarship Council. 
${ }^{1}$ D. Rockwell and E. Naudascher, "Self-sustained oscillations of impinging free shear layers," Annu. Rev. Fluid Mech. 11, 67-94 (1979).

${ }^{2}$ D. Rockwell, J.-C. Lin, P. Oshkai, M. Reiss, and M. Pollack, "Shallow cavity flow tone experiments: Onset of locked-on states," J. Fluid Struct. 17, 381-414 (2003).

${ }^{3}$ S. C. Morris, "Shear-layer instabilities: Particle image velocimetry measurements and implications for acoustics," Annu. Rev. Fluid Mech. 43, 529-550 (2011).

${ }^{4}$ B. L. O'Connor, M. Hondzo, and J. W. Harvey, "Predictive modeling of transient storage and nutrient uptake: Implications for stream restoration," J. Hydraul. Eng. 136, 1018-1032 (2010).

${ }^{5}$ T. R. Jackson, R. Haggerty, S. V. Apte, A. Coleman, and K. J. Drost, "Defining and measuring the mean residence time of lateral surface transient storage zones in small streams," Water Resour. Res. 48, W10501, doi:10.1029/2012wr012096 (2012).

${ }^{6}$ T. R. Jackson, R. Haggerty, S. V. Apte, and B. L. O'Connor, “A mean residence time relationship for lateral cavities in gravel-bed rivers and streams: Incorporating streambed roughness and cavity shape," Water Resour. Res. 49, 1-9, doi:10.1029/2012WR012444 (2013).

${ }^{7}$ J. C. F. Pereira and J. M. M. Sousa, "Experimental and numerical investigation of flow oscillations in a rectangular cavity," J. Fluids Eng. 117, 68-74 (1995).

${ }^{8}$ S. Bian, J. F. Driscoll, B. R. Elbing, and S. L. Ceccio, "Time resolved flow-field measurements of a turbulent mixing layer over a rectangular cavity,” Exp. Fluids 51, 51-63 (2011).

${ }^{9}$ B. Tuna, E. Tinar, and D. Rockwell, "Shallow flow past a cavity: Globally coupled oscillations as a function of depth," Exp. Fluids 54, 1586 (2013).

${ }^{10}$ X. Liu and J. Katz, "Vortex-corner interactions in a cavity shear layer elucidated by time-resolved measurements of the pressure field," J. Fluid Mech. 728, 417-457 (2013).

${ }^{11}$ M. Sanjou and I. Nezu, "Hydrodynamic characteristics and related mass-transfer properties in open-channel flows with rectangular embayment zone," Environ. Fluid Mech. 13, 527-555 (2013).

12 W. Uijttewaal, D. Lehmann, and A. van Mazijk, "Exchange processes between a river and its groyne fields: Model experiments," J. Hydraul. Eng. 127, 928-936 (2001).

${ }^{13}$ A. McCoy, G. Constantinescu, and L. J. Weber, "Numerical investigation of flow hydrodynamics in a channel with a series of groynes," J. Hydraul. Eng. 134, 157-172 (2008).

14 V. Weitbrecht, S. Socolofsky, and G. Jirka, "Experiments on mass exchange between groyne fields and main stream rivers," J. Hydraul. Eng. 134, 173-183 (2008).

${ }^{15}$ M. Wolfinger, C. A. Ozen, and D. Rockwell, "Shallow flow past a cavity: Coupling with a standing gravity wave," Phys. Fluids 24, 104103 (2012).

${ }^{16}$ E. Mignot, C. Zeng, G. Dominguez, C. Li, N. Riviere, and P. Bazin, "Impact of topographic obstacles on the discharge distribution in open-channel bifurcations," J. Hydrol. 494, 10-19 (2013).

${ }^{17}$ E. Mignot, D. Doppler, N. Riviere, I. Vinkovic, J. Gence, and S. Simoens, "Analysis of flow separation using a local frame-axis: Application to the open-channel bifurcation," J. Hydraul. Eng. 140, 280-290 (2014).

${ }^{18}$ M. Jahanmiri, "Particle image velocimetry: Fundamentals and its application," Report 2011:03, ISSN: 1652-8549 Chalmers University of Technology, Gteborg, Sweden, 2011.

${ }^{19}$ R. Booij, "Exchange of mass in harbours," in 23rd Congress of IAHR, Ottawa: D69-D74 (National Research Council of Canada, Ottawa, 1989).

${ }^{20}$ I. Kimura and T. Hosoda, "Fundamental properties of flows in open channel with dead zone," J. Hydraul. Eng. 123(2), 98-107 (1997).

${ }^{21}$ K. Mizumura and M. Yamasaka, "Flow in open-channel embayments," J. Hydraul. Eng. 128, 1098-1101 (2002)

${ }^{22}$ S. Pope, Turbulent Flows (Cambridge University Press, 2008).

${ }^{23}$ P. A. Durbin and B. A. Pettersson-Reif, Statistical Theory and Modeling for Turbulent Flows, 2nd ed. (Wiley, 2010).

${ }^{24}$ S. Proust, J. Fernandes, Y. Peltier, J. Leal, N. Riviere, and A. Cardoso, "Turbulent non-uniform flows in straight compound open-channels," J. Hydraul. Res. 51, 656-667 (2013).

${ }^{25}$ M. Raupach, J. Finnigan, and Y. Brunet, "Coherent eddies and turbulence in vegetation canopies: The mixing-layer analogy," Boundary Layer Meteorol. 78, 351-382 (1996).

${ }^{26}$ M. Lesieur, Turbulence in Fluids (Kluwer Academic, Dordrecht, 1997).

${ }^{27}$ L. Graftieaux, M. Michard, and N. Grosjean, "Combining PIV, POD and vortex identification algorithms for the study of unsteady turbulent swirling flows," Meas. Sci. Technol. 12, 1422-1429 (2001).

${ }^{28}$ R. Shaw, Y. Brunet, J. Finnigan, and M. Raupach, "A wind tunnel study of air flow in waving wheat: Two-point velocity statistics," Boundary Layer Meteorol. 76, 349-376 (1995).

${ }^{29}$ C. Zhang, R. Shaw, and U. Paw, "Spatial characteristics of turbulent coherent structures within and above an orchard canopy," in Precipitation Scavenging and Atmosphere-Surface Exchange, edited by S. E. Schwartz and W. G. N. Slinn (Hemisphere Publishing Co., Washington, 1992), pp. 741-751.

${ }^{30}$ P. Holmes, J. L. Lumley, and G. Berkooz, Turbulence, Coherent Structures, Dynamical Systems and Symmetry (Cambridge University Press, Cambridge, 1996).

${ }^{31}$ B. Silverman, Density Estimation for Statistics and Data Analysis, Monographs on Statistics and Applied Probability (Chapman and Hall, London, 1986).

${ }^{32}$ E. Valentine and I. Wood, “Longitudinal dispersion with dead zones,” J. Hydraul. Div., ASCE 103, 975-990 (1977). 